\title{
1 Resonance-enhanced terahertz nanoscopy probes
}

2 Thomas Siday ${ }^{* \dagger}$, Lucy L Hale, Rodolfo I Hermans and Oleg Mitrofanov*

3 University College London, London, UK

4 KEYWORDS near-field microscopy, s-SNOM, nano-spectroscopy, resonant scattering.

5 ABSTRACT Near-field nanoscopy at terahertz $(\mathrm{THz})$ frequencies is uniquely placed to probe a

6 multitude of physical phenomena at the nanoscale. However, at $\mathrm{THz}$ frequencies, the scattering

7 efficiency of standard near-field probes is poor, limiting the sensitivity, and hence resolution of

8 the technique. Here, we propose and demonstrate tunable resonant scattering from metal tips which

9 allow us to overcome this limitation. Tips supporting a $\lambda / 2$ dipolar resonance in the $\mathrm{THz}$ range are

10 fabricated from indium metal directly on the tine of a quartz tuning fork. We observe enhancement

11 of the THz scattering efficiency at the resonance frequency with Q-factor of $\sim 2-3$. These tips

12 enable a subwavelength spatial resolution better than $100 \mathrm{~nm}$. We support the experimentally

13 observed enhancement using a numerical model. The enhanced scattering efficiency afforded by

14 the resonant indium tips can enable the probing of new phenomena, such as plasmons in 2-

15 dimensional materials which have proven difficult to observe thus far. 
2 TOC GRAPHIC
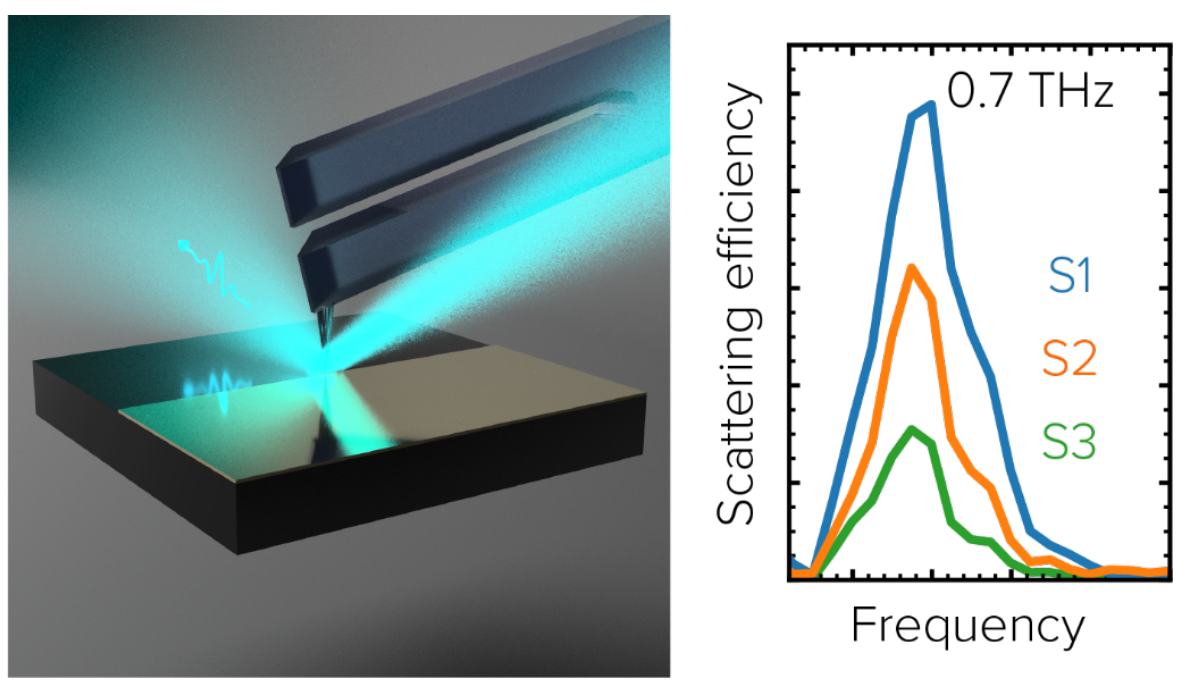

4 Near-field nanoscopy has enabled direct probing of a variety of phenomena which are difficult

5 or impossible to access with far-field techniques, including polaritons in 2-dimensional materials ${ }^{1}$,

6 particularly graphene plasmons ${ }^{2-5}$ and phonons in hexagonal boron nitride ${ }^{6,7}$, alongside modes

7 within individual subwavelength resonators ${ }^{8,9}$, and even single quantum dots ${ }^{10}$ and molecules ${ }^{11}$.

8 Whilst in the infrared and visible ranges, near-field microscopy has become a powerful scientific

9 tool, at terahertz $(\mathrm{THz})$ frequencies $(0.3-5 \mathrm{THz})$, application of near-field nanoscopy ${ }^{12,13}$ is still

10 limited due to poor scattering efficiency. This is despite recent instrumentation improvements

11 including self-mixing detection using a quantum cascade laser (QCL) ${ }^{14-16}$, the use of quartz tuning

12 forks ${ }^{16-18}$, and direct detection of fields at the tip apex ${ }^{4}$. However, only a few works have addressed

13 the poor efficiency of the scattering process itself, the central limitation to the sensitivity of near-

14 field nanoscopy techniques. Whilst coupling of the incident wave to the tip apex can be improved

15 with a grating coupler ${ }^{19}$, photonic crystal $^{20}$, and radially polarized light sources ${ }^{21}$, these approaches

16 often require elaborate fabrication methods. Recently, a simpler alternative has been proposed: a

17 scattering probe acting as a resonant dipole antenna ${ }^{21-23}$. This can improve both the efficiency of 
1 coupling to and scattering from the tip. However, it has proved difficult to implement it in practice,

2 because controlling resonance frequency requires fine control over the tip geometry. Mastel et al.

3 fabricated THz resonant tips by machining gold wire with high precision using a focused ion beam

$4 \quad(\text { FIB })^{23}$. Such tips are unfortunately not easily accessible to most researchers, whereas the use of

5 commercial tips, which can support resonances at $\mathrm{THz}$ frequencies, are hindered by lack of precise

6 control over the geometry. Recently, we demonstrated that it is possible to fabricate indium cones

7 supporting dipolar resonances at $\mathrm{THz}$ frequencies using a remarkably simple fabrication process ${ }^{21}$.

8 In this work, we demonstrate that the indium tips can enhance the scattering process in $\mathrm{THz}$ near-

9 field microscopy. We achieve control over the tip geometry, and thus resonant enhancement of the

10 scattering efficiency in the $\mathrm{THz}$ frequency range through careful control of the tip fabrication

11 parameters, allowing us to fabricate tips with length from $30 \mu \mathrm{m}$ to over $1 \mathrm{~mm}$, with apex diameter

12 on the order of $50 \mathrm{~nm}^{21}$. We find the resonant probes exhibit enhancement of the scattered field

13 with a quality factor between 2 and 3, in the first as well as in second and third demodulation

14 harmonics, alongside nanoscale field confinement at the tip apex. For sub-nanometer control over

15 the distance between the tip and sample we use a quartz tuning fork ${ }^{24}$. We anticipate this resonant

16 enhancement of scattering by the tip will push the sensitivity limits of THz near-field nanoscopy,

17 and will enable applications for systems still inaccessible due to limitations in signal to noise ratio

18 (SNR), leading to new physical insights. 

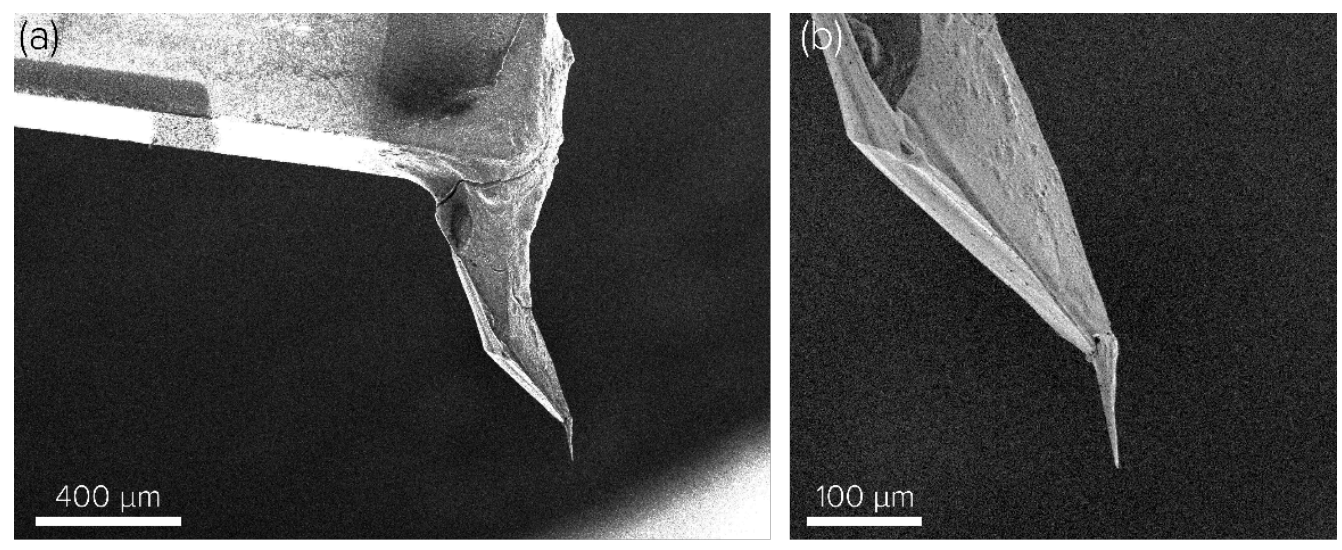

(C)

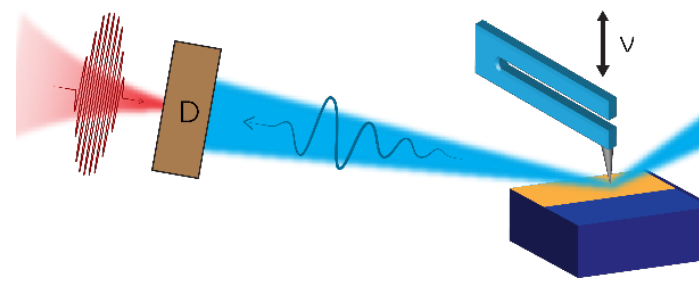

2 Figure 1. Resonant scattering probes for $\mathrm{THz}$ nano-spectroscopy. (a, b) Scanning electron

3 micrograph (SEM) image of a $100 \mu \mathrm{m}$ long indium tip mounted to the tine of a quartz tuning fork.

4 (c) Schematic of the $\mathrm{THz}$ near-field microscope. Coherent $\mathrm{THz}$ pulses are generated through

5 optical rectification of near infra-red pulses in a ZnTe crystal (S). Parabolic mirrors (PM) collimate

6 and focus the THz pulses onto the scattering tip, which is modulated normal to the sample surface

7 at frequency $\nu$. THz fields scattered by the tip are detected using a photoconductive detector.

\section{RESULTS AND DISCUSSION}

\section{$9 \quad$ Near-field nanoscopy with resonant indium tips}

10 For nanoscale resolution we need to control the position of the scattering probe over a sample

11 surface with sub-nanometer accuracy. Whilst silicon cantilever probes for atomic force

12 microscopy (AFM) are available (e.g. Neaspec, $\mathrm{GmbH})$, they are not practical for accommodating 
1 relatively large resonant structures, such as the indium tips. Instead, we use a quartz tuning fork,

2 which can sense the atomic force and provide the required control over the tip-sample

3 separation ${ }^{24,25}$. Tuning forks with sharp needle probes have already been applied to $\mathrm{THz}$

4 nanoscopy ${ }^{16-18}$, and provide a compelling alternative to the conventional AFM cantilever due to

5 their self-sensing properties, and high force sensitivity ${ }^{24}$. Here, instead of the needle, we use the

6 much smaller indium tip (length $\sim 50-100 \mu \mathrm{m}$ ), which can support dipolar resonances at $\mathrm{THz}$

7 frequencies. A scanning electron micrograph (SEM) image of a $100 \mu \mathrm{m}$ long indium tip mounted

8 to the tine of the quartz tuning fork is shown in Figure 1(a, b). To ensure the incident THz pulse is

9 not deflected by the tuning fork before reaching the tip, a shard of quartz is attached to one tine of

10 the tuning fork before mounting the tip (supporting information section A1). We modulate the

11 position of the quartz tuning fork normal to the surface of a sample at the resonant frequency of

12 the tuning fork $(v \sim 32 \mathrm{kHz})$, enabling the required sub-nanometer tip-sample distance control ${ }^{24,25}$.

13 To prevent measurement artefacts emerging due to variations in the tapping amplitude, we also

14 continuously maintain the tapping amplitude of the tip at $\sim 100 \mathrm{~nm}$.

15 To evaluate the scattering efficiency enhancement of the resonant indium tips we illuminate the

16 tip with a broadband $\mathrm{THz}$ pulse and detect the electric field of the tip-scattered pulse using the

17 experimental configuration shown in Figure 1(c). In our configuration, we can position the same

$18 \mathrm{THz}$ detector in the place of the tuning fork and thus directly measure the pulse incident on the tip.

19 We then can obtain the scattering efficiency spectrum directly by normalizing the scattered field

20 spectrum to the incident field spectrum. Since the tip-scattered field amplitude depends non-

21 linearly on the sample probe separation, in order to extract the tip-scattered wave from the

22 background field we demodulate the signal at the tip tapping frequency $v$, and its higher 23 harmonics $^{26}$. 
1 To excite the tip resonance, we use broadband $\mathrm{THz}$ pulses generated via optical rectification in

2 a ZnTe crystal of $100 \mathrm{fs}$ long near infra-red pulses $(\lambda=800 \mathrm{~nm})$ from a Ti:Sapphire oscillator. The

$3 \mathrm{THz}$ pulses are collimated and re-focused using two parabolic mirrors onto the tip and sample at

4 an angle of $30^{\circ}$ (Fig. 1(c)). We use a photoconductive detector with a plasmonic optical

5 metasurface ${ }^{27}$ to detect the $\mathrm{THz}$ pulse waveforms in the time domain so we can access both the

6 relative amplitude and the phase of the scattered field. We note that this photoconductive detector

7 does not use a lens to capture the scattered THz field. Whilst the lens can improve the collection

8 efficiency of the detector, it can also introduce errors in measuring the scattered and incident field

9 due to lens alignment. Our simple experimental configuration on the other hand, allows us to

10 evaluate the THz field scattered by the tip quantitatively, albeit at the expense of sensitivity.

11 (supporting information, section A2).

(a)

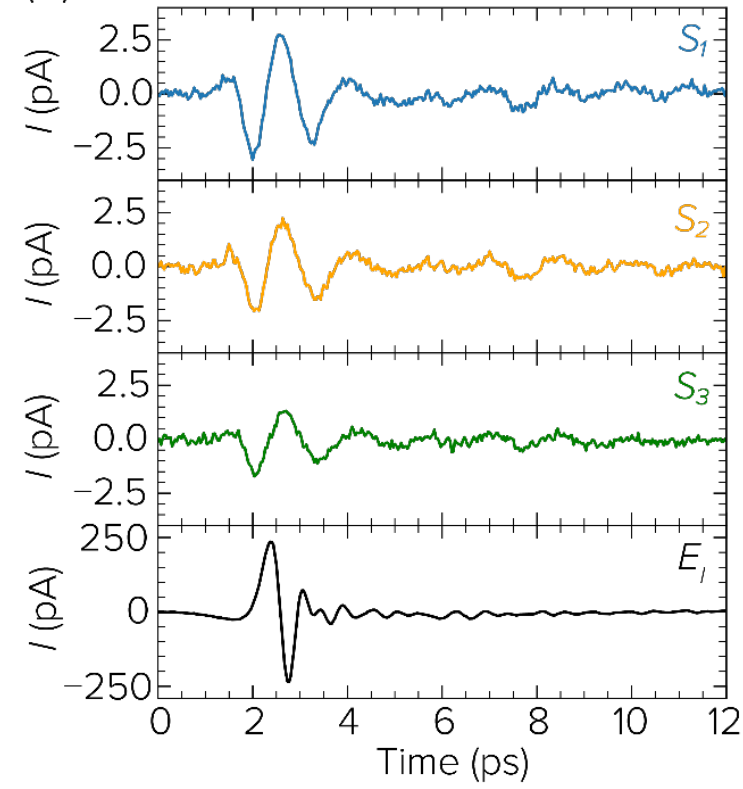

(b)

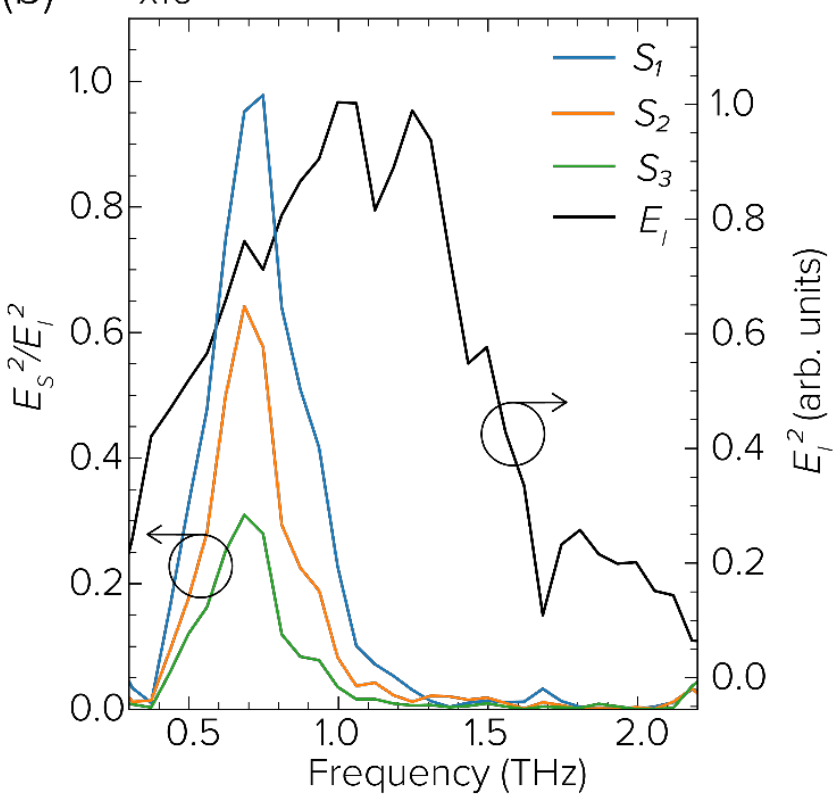

13 Figure 2. Scattering from a $100 \mu \mathrm{m}$ long resonant indium tip. (a) Scattered THz pulse waveform,

14 measured at the first, S1 (blue), second, S2 (orange) and third, S3 (green) harmonic of the fork

15 tapping frequency. The THz waveforms are measured with a lock-in filter constant of $300 \mathrm{~ms}$, and 
1 are averaged five times. The black curve shows the THz pulse incident on the indium tip. (b)

2 Fourier transform of the waveforms in (a) normalized to the incident $\mathrm{THz}$ pulse spectrum. The 3 waveforms are cropped at $\tau \sim 7$ ps to eliminate a pulse reflection from the analysis. The uncropped 4 spectra are provided in the supporting information, section A3.

5 Waveforms of the incident and scattered $\mathrm{THz}$ pulses for the $100 \mu \mathrm{m}$ long indium tip are shown 6 in Figure 2(a). The tip-scattered pulse can be extracted by demodulating at the tapping frequency 7 of the tuning fork $(v \sim 32 \mathrm{kHz})$, which we denote as S1. Since S1 may contain some background 8 signals we also demodulate at the second (S2) and third (S3) harmonic of the tapping frequency.

9 The normalized scattering efficiency spectrum of the $100 \mu \mathrm{m}$ long indium tip is shown in 10 Figure 2(b). We see a clear peak in scattered field spectrum indicating enhancement of the 11 scattered field at the tip resonance frequency. All three demodulation harmonics show 12 enhancement at the same frequency.

13 In order to confirm that the spectral enhancement corresponds to the dipole resonance along the 14 shaft of the indium tip, we simulate the structure using a commercially available time-domain 15 solver (CST studio suite) closely replicating the experimental conditions: a $100 \mu \mathrm{m}$ long metal 16 cone with an apex radius of $50 \mathrm{~nm}$ is positioned above a uniform metal surface (Fig. 3(a)); the tip 17 is excited with a broadband $\mathrm{THz}$ pulse at an angle of $30^{\circ}$ to the metal plane. We evaluate the $\mathrm{THz}$ 18 field near the tip apex for a tip-sample separation of $10 \mathrm{~nm}$, which we use to represent the distance 19 of closest approach for the tip. We see two clear peaks in the normalized spectra (Fig. 3(b)), at $20 \quad 0.9 \mathrm{THz}$ and $2.05 \mathrm{THz}$, corresponding to two lowest frequency resonances. Figure 3(c) shows the 21 magnitude of the electric field on a cross-section of the tip at $0.9 \mathrm{THz}$. This is strikingly similar to 22 the half-wave dipole $(\lambda / 2)$ dipole resonance ${ }^{28}$, in agreement with previous near-field studies of $\mathrm{THz}$ 23 resonant scattering probes ${ }^{21-23}$. The frequency of the resonance agrees reasonably well with the 
1 scattering efficiency measurements for a tip of the same length (Fig. 2(b)), confirming that the

2 enhancement observed in the experiments is indeed due to the dipolar resonance along the tip shaft.

3 We do not observe the high-frequency peak centered at $2.05 \mathrm{THz}$ in our experiment. We explain

4 this by considering the antenna properties of the scattering tip. Figure 3(d) shows the magnitude

5 of the electric field on a cross-section of the tip at $2.05 \mathrm{THz}$. Here, the field distribution on the tip

6 is different. Whilst we also see a dipolar mode along the tip shaft, it bears most similarity to the

7 full wave $(\lambda)$ resonance $^{28}$, providing a possible explanation for the absence of the high frequency

8 peak in our experimental measurements: whilst the half wave $(\lambda / 2)$ dipole mode couples well to

9 the far-field, the full wave $(\lambda)$ resonance does not, at least in the case of the ideal dipole ${ }^{28}$. The

10 poor coupling efficiency of the full-wave resonance of the indium tips, coupled with the low SNR

11 our experiment frequencies above $1.7 \mathrm{THz}$ means that the full wave resonance is not observed.
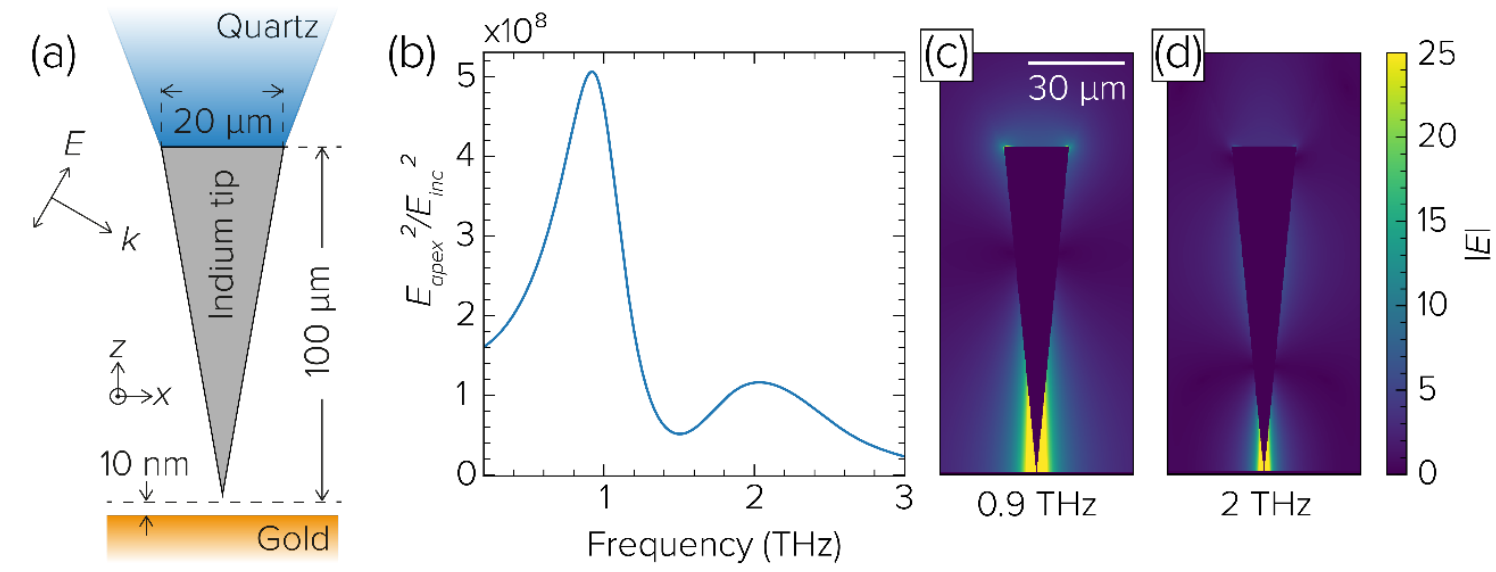

13 Figure 3. Numerical modelling of resonant scattering probes. (a) schematic showing the

14 configuration of the numerical model and geometry of the metal cone. (b) Intensity of the electric

15 field polarized along the z-axis, normalized to the incident $\mathrm{THz}$ pulse, and evaluated directly

16 between the cone apex and metal surface shown in (a). (c,d) Magnitude of the electric field on the

17 metal cone at (c) $0.9 \mathrm{THz}$ and (d) $2.05 \mathrm{THz}$. To highlight the electric field distribution induced by 
1 the tip resonance, the color scale is limited to a field enhancement of 25. Another version of this

2 figure without saturation of the color scale is provided in supporting information section A4.

\section{Controlling the resonance frequency and bandwidth.}

4 The dipolar resonance of the indium tip shown in Figure 2 covers only a fraction of the available

$5 \mathrm{THz}$ spectrum. We showed previously that the frequency of the tip resonance depends sensitively

6 on the tip length ${ }^{21}$, and we can use this sensitivity to enhance the scattering efficiency for a desired

7 frequency range. We found that by carefully controlling the fabrication parameters (see supporting

8 information section A1), it is possible to fabricate tips of a given length. To demonstrate tuning

9 the tip resonance, we fabricate two similar tips, in this case with length of $60 \mu \mathrm{m}$ and $65 \mu \mathrm{m}$. Figure

10 4(a) shows SEM images of the new tips, alongside the $100 \mu \mathrm{m}$ long tip already discussed. To

11 ensure the conditions for scattering were not significantly varied, the same quartz tuning fork, gold

12 surface and tapping amplitude was used for each indium tip. Since the variation in tip mass is small

13 in comparison to the mass of the tuning fork, altering the length of the indium tip does not

14 significantly alter the force sensing properties of the tuning fork. Waveforms of the THz pulses

15 scattered by each tip at three harmonics of the tip tapping frequency $v$ are shown in Figure 4(b),

16 and Figure 4(c) shows the scattering efficiency for each of the tips. Measured at the tip tapping

17 frequency (S1), the resonant frequency of the tips is $0.72 \mathrm{THz}$ for the $100 \mu \mathrm{m}$ tip, $1 \mathrm{THz}$ for the

$1865 \mu \mathrm{m}$ long tip, and $1.05 \mathrm{THz}$ for the $60 \mu \mathrm{m}$ long tip. In the second and third harmonics. The

19 resonant frequency of each tip varies by less than $50 \mathrm{GHz}$ across the three harmonics. 

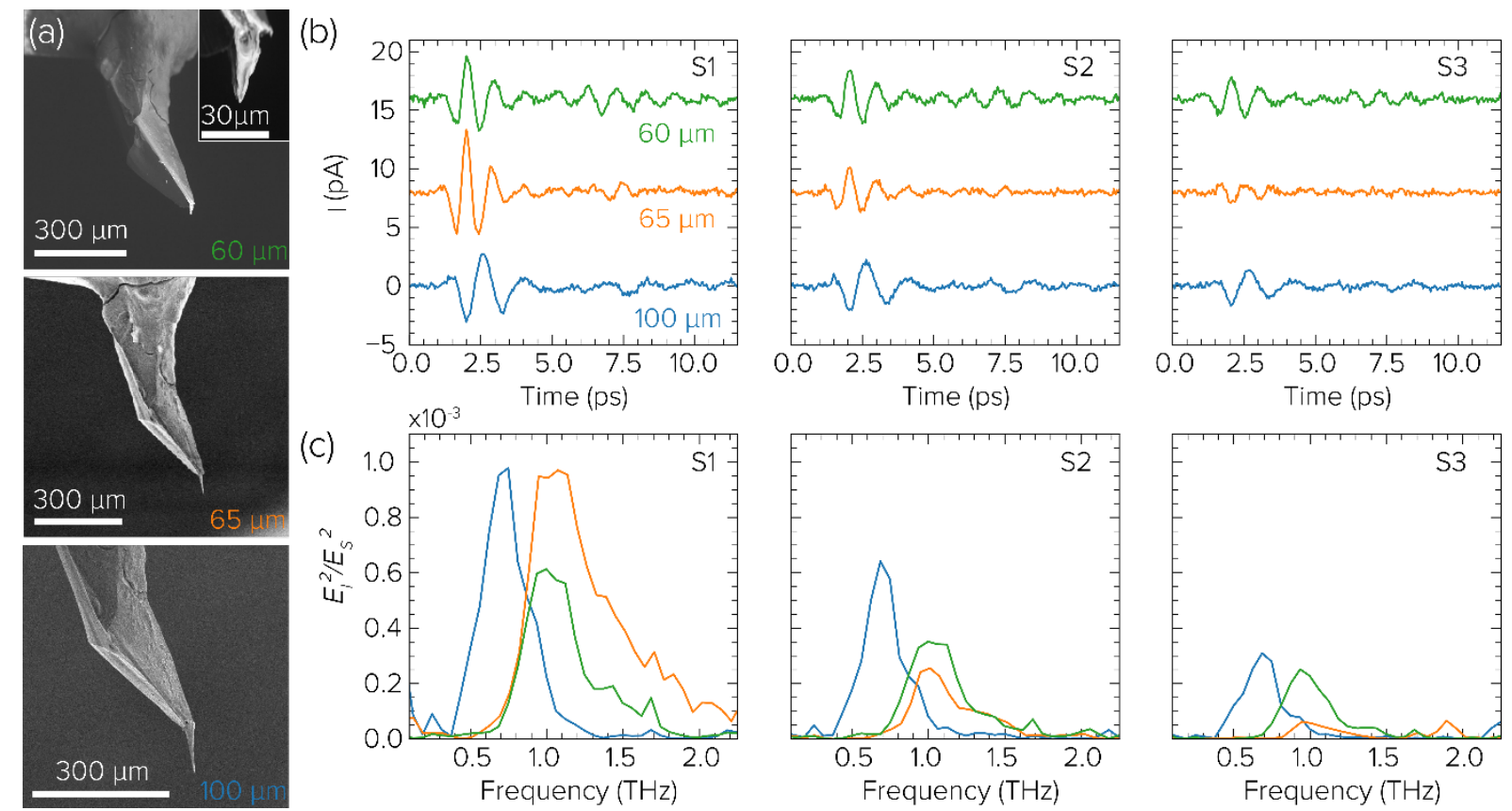

2 Figure 4. Tuning the tip resonant frequency by altering the tip length. (a) SEM images the measured tips, $60 \mu \mathrm{m}$ (upper), $65 \mu \mathrm{m}$ (center) and $100 \mu \mathrm{m}$ (lower). Note that the SEM image of

4 the $60 \mu \mathrm{m}$ long tip was taken at an oblique angle, reducing the apparent length of the tip. (b) THz

5 pulses scattered by the three tips at the first harmonic, S1 (left panel), second harmonic, S2 (center

6 panel) and third harmonic, S3 (right hand panel) of the tip tapping frequency $v$. (c) Normalized

7 scattering efficiency spectra for the three tips at S1 (left), S2 (center) and S3 (right).

Field confinement at the tip apex

9 The crucial tip property for achieving nanoscale spatial resolution in near-field microscopy is

10 the ability to spatially confine the electric field at the tip apex. The tip apex for the indium tips,

11 evaluated directly from SEM images, was on the order of $50 \mathrm{~nm}^{21}$. To confirm the spatial

12 resolution of the indium tips experimentally, we measure THz pulses scattered from the tip whilst

13 increasing the separation between the tip apex and gold sample. A constant tapping amplitude was

14 maintained while the tip-sample separation was increased. Figure 5(a) shows the THz field 15 scattered by the tip at the tapping frequency, $v(\mathrm{~S} 1)$, measured at the peak of the THz pulse 
1 (Fig. 5(b)). As the tip-sample separation reaches approximately $100 \mathrm{~nm}$, the scattered field 2 amplitude falls by 5-10 times, below the noise floor. By fitting an exponential function, we 3 estimate the 1/e field confinement is on the order of $50 \mathrm{~nm}$. For the second harmonic (S2), the

4 decay occurs over a shorter length scale, with the scattered field falling below the noise floor in 5 less than $50 \mathrm{~nm}$. This increased spatial confinement at higher demodulation orders of the tapping

6 frequency is in good accordance with previous observations and demonstrates the nanoscale field 7 confinement required for near-field imaging with high spatial resolution. We also measured the 8 amplitude of the $\mathrm{THz}$ field scattered by the tip as it is moved across a metal-dielectric interface 9 (Fig. 5(c)). Here, we also see spatial resolution better than $100 \mathrm{~nm}$.
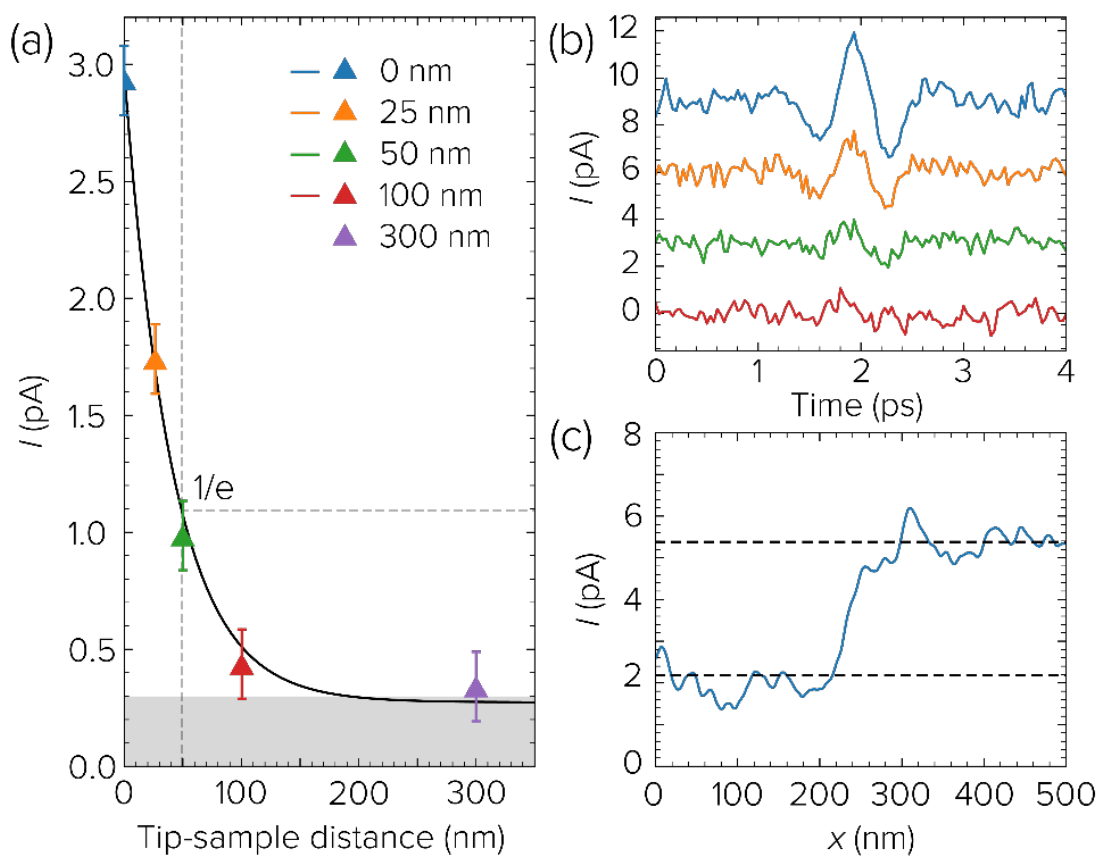

11 Figure 5. Field confinement at the apex of the indium tip. (a) Electric field measured at the peak

12 of the THz pulse in (b). The black curve is an exponential function fitted to the data. The grey

13 dashed lines indicate the distance at which the exponential function decays by a factor of $1 / \mathrm{e}$. The

14 grey region indicates the noise floor of the measurement. Note that the tip-sample distance $0 \mathrm{~nm}$ 15 refers to when the AFM feedback loop is engaged. For larger distances, the feedback loop is 
1 disengaged, and the tip-sample distance is increased by retracting the sample. (b) THz pulses

2 scattered by the tip for increasing tip-sample distance. (c) Amplitude of the scattered field

3 measured at the peak of the $\mathrm{THz}$ pulse, as the indium tip is scanned across a metal-dielectric

4 interface.

5 The nanometer scale field confinement and the spectral enhancement obtained with the indium

6 tips are the key requirements for improving SNR in THz near-field microscopy without sacrificing

7 spatial resolution. A typical linewidth of indium tips in this work is on the order of $0.3-0.5 \mathrm{THz}$,

8 with a Q-factor between 2 and 3 (Fig. 4), consistent with Q-factors was measured previously for

9 indium cones of similar geometry ${ }^{21}$. For near-field microscopy applications, the linewidth is

10 sufficiently wide to perform $\mathrm{THz}$ spectroscopy analysis using short $\mathrm{THz}$ pulses. The same tip

11 geometry can be also used to enhance the SNR for single-frequency measurements employing $\mathrm{THz}$

12 sources, such as QCLs, non-linear mixers and gas lasers.

13 The resonance frequency can be easily tuned by varying the tip length. The Q-factor in principle

14 also can be changed by altering the cone angle of the indium tip ${ }^{21}$. We anticipate that by controlling

15 the size of base for the tip mount, it will be possible to tune both the linewidth and resonant

16 frequency of the indium tips. It is also important to note that the field confinement at the apex is

17 similar to, or better than many recent demonstrations of the spatial resolution of nanoscopy probes

18 in combination with broadband $\mathrm{THz}$ sources ${ }^{17,29}$. The scattering efficiency is strongly dependent

19 on the radius of the tip apex: a sharper apex decreases the scattering efficiency while enabling a

20 better field confinement. Therefore, any gain in SNR can be translated into higher spatial 21 resolution.

22 The indium tips are not dependent on any individual measurement scheme, since they directly 23 improve the fundamental limitation of all scattering probes: the scattering efficiency. Therefore, 
1 these tips can serve as a drop-in replacement for any tuning-fork based $\mathrm{THz}$ nanoscopy technique.

2 As a final note regarding SNR, we emphasize that this system used a $\mathrm{THz}$ detector with a relatively

3 low numerical aperture (a photoconductive detector with no lens). By incorporating more efficient

4 collection optics, such as a pair of parabolic mirrors, the SNR could be significantly improved.

5 CONCLUSION

6 In summary, for the first time we have demonstrated resonant enhancement of scattering from

7 THz near-field scattering probe. We achieved this using a sharp indium tip which supports a $\lambda / 2$

8 dipolar resonance, mounted to the tine of a quartz tuning fork. We directly evaluated scattering

9 efficiency spectra for tips of different length using broadband $\mathrm{THz}$ time-domain spectroscopy. The

10 quartz tuning fork enables sub-nanometer control over the tip-sample separation, and we

11 demonstrate $\mathrm{THz}$ field confinement by the tip apex, which enables spatial resolution better than

$12100 \mathrm{~nm}$. These tunable resonant scattering tips will enable a wide range of new studies at $\mathrm{THz}$

13 frequencies using both broadband and single-frequency measurement schemes and could push the

14 present resolution and sensitivity limits in the $\mathrm{THz}$ frequency range.

SUPPORTING INFORMATION

17 Supporting Information Available: A description of the fabrication process for the indium tips is

18 provided in supporting section A1. Section A2 provides a detailed description of the electronics

19 required for sub-nanometer control over the tip-sample separation, and THz TDS configuration

20 used to generate and detect THz pulses. Section A3 shows the normalized scattering spectra for

21 each tip for the full THz transients, including the reflection seen after 7 ps. Section A4 shows the

22 electric field on a cross section of the tip without saturating the color scale used in Figure 3(c,d).

23 This material is available free of charge via the internet at http://pubs.acs.org 
2 AUTHOR INFORMATION

3 Corresponding Author

$4 \quad$ *tom.siday@ur.de

$5 \quad$ *o.mitrofanov@ucl.ac.uk

6 Present Addresses

$7 \quad †$ Department of Physics, University of Regensburg, 93040 Regensburg, Germany

8 Author Contributions

9 T.S designed and built the experiment, T.S. and L.H. acquired measurements of the tip scattering

10 efficiency, R.H contributed to experimental design. O.M. fabricated the photoconductive

11 detectors. All authors contributed to the discussion of the results and to the editing of the

12 manuscript. O.M. coordinated the overall research.

\section{ACKNOWLEDGMENT}

14 This work was supported by the EPSRC (EP/L015277/1, EP/P021859/1, EP/L015455/1) and by

15 the U.S. Department of Energy, Office of Basic Energy Sciences, Division of Materials Sciences 16 and Engineering. $\mathrm{THz}$ detector fabrication was performed at the Center for Integrated

17 Nanotechnologies, an Office of Science User Facility operated for the U.S. Department of Energy

18 (DOE) Office of Science. Sandia National Laboratories is a multimission laboratory managed and

19 operated by National Technology and Engineering Solutions of Sandia, LLC., a wholly owned

20 subsidiary of Honeywell International, Inc., for the U.S. Department of Energy's National Nuclear

21 Security Administration under contract DE-NA-0003525. 


\section{REFERENCES}

(1) Basov, D. N.; Fogler, M. M.; García de Abajo, F. J. Polaritons in van Der Waals Materials. Science 2016, 354 (6309), aag1992. https://doi.org/10.1126/science.aag1992.

(2) Fei, Z.; Rodin, A. S.; Andreev, G. O.; Bao, W.; McLeod, A. S.; Wagner, M.; Zhang, L. M.; Zhao, Z.; Thiemens, M.; Dominguez, G.; et al. Gate-Tuning of Graphene Plasmons Revealed by Infrared Nano-Imaging. Nature 2012, 487 (7405), 82-85. https://doi.org/10.1038/nature11253.

(3) Mitrofanov, O.; Yu, W.; Thompson, R. J.; Jiang, Y.; Greenberg, Z. J.; Palmer, J.; Brener, I.; Pan, W.; Berger, C.; De Heer, W. A.; et al. Terahertz Near-Field Imaging of Surface Plasmon Waves in Graphene Structures. Solid State Commun. 2015, 224, 47-52. https://doi.org/10.1016/j.ssc.2015.08.013.

(4) Alonso-González, P.; Nikitin, A. Y.; Gao, Y.; Woessner, A.; Lundeberg, M. B.; Principi, A.; Forcellini, N.; Yan, W.; Vélez, S.; Huber, A. J.; et al. Acoustic Terahertz Graphene Plasmons Revealed by Photocurrent Nanoscopy. Nat. Nanotechnol. 2016, 12 (1), 31-35. https://doi.org/10.1038/nnano.2016.185.

(5) Ni, G. X.; McLeod, A. S.; Sun, Z.; Wang, L.; Xiong, L.; Post, K. W.; Sunku, S. S.; Jiang, B.-Y.; Hone, J.; Dean, C. R.; et al. Fundamental Limits to Graphene Plasmonics. Nature 2018, 557 (7706), 530-533. https://doi.org/10.1038/s41586-018-0136-9.

(6) Yoxall, E.; Schnell, M.; Nikitin, A. Y.; Txoperena, O.; Woessner, A.; Lundeberg, M. B.; Casanova, F.; Hueso, L. E.; Koppens, F. H. L.; Hillenbrand, R. Direct Observation of Ultraslow Hyperbolic Polariton Propagation with Negative Phase Velocity. Nat. Photonics 2015, 9 (10), 674-678. https://doi.org/10.1038/nphoton.2015.166.

(7) Shi, Z.; Bechtel, H. A.; Berweger, S.; Sun, Y.; Zeng, B.; Jin, C.; Chang, H.; Martin, M. C.; Raschke, M. B.; Wang, F. Amplitude- and Phase-Resolved Nanospectral Imaging of Phonon Polaritons in Hexagonal Boron Nitride. ACS Photonics 2015, 2 (7), 790-796. https://doi.org/10.1021/acsphotonics.5b00007.

(8) Rang, M.; Jones, A. C.; Zhou, F.; Li, Z.-Y.; Wiley, B. J.; Xia, Y.; Raschke, M. B. Optical 
Near-Field Mapping of Plasmonic Nanoprisms. Nano Lett. 2008, 8 (10), 3357-3363. https://doi.org/10.1021/nl801808b.

(9) Hale, L. L.; Keller, J.; Siday, T.; Hermans, R. I.; Haase, J.; Reno, J. L.; Brener, I.; Scalari, G.; Faist, J.; Mitrofanov, O. Non-Invasive Near-Field Spectroscopy of Single SubWavelength Complementary Resonators. Laser Photonics Rev. (under reveiw).

(10) Jacob, R.; Winnerl, S.; Fehrenbacher, M.; Bhattacharyya, J.; Schneider, H.; Wenzel, M. T.; Ribbeck, H.-G. von; Eng, L. M.; Atkinson, P.; Schmidt, O. G.; et al. Intersublevel Spectroscopy on Single InAs-Quantum Dots by Terahertz Near-Field Microscopy. Nano Lett. 2012, 12 (8), 4336-4340. https://doi.org/10.1021/nl302078w.

(11) Cocker, T. L.; Peller, D.; Yu, P.; Repp, J.; Huber, R. Tracking the Ultrafast Motion of a Single Molecule by Femtosecond Orbital Imaging. Nature 2016, 539 (7628), 263-267. https://doi.org/10.1038/nature19816.

(12) Mastel, S.; Govyadinov, A. A.; Maissen, C.; Chuvilin, A.; Berger, A.; Hillenbrand, R. Understanding the Image Contrast of Material Boundaries in IR Nanoscopy Reaching $5 \mathrm{Nm}$ Spatial Resolution. ACS Photonics 2018, 5 (8), 3372-3378. https://doi.org/10.1021/acsphotonics.8b00636.

(13) Maissen, C.; Chen, S.; Nikulina, E.; Govyadinov, A.; Hillenbrand, R. Probes for Ultrasensitive $\mathrm{THz}$ Nanoscopy. ACS Photonics 2019, 6 (5), 1279-1288. https://doi.org/10.1021/acsphotonics.9b00324.

(14) Dean, P.; Mitrofanov, O.; Keeley, J.; Kundu, I.; Li, L.; Linfield, E. H.; Giles Davies, A. Apertureless Near-Field Terahertz Imaging Using the Self-Mixing Effect in a Quantum Cascade Laser. Appl. Phys. Lett. 2016, 108 (9), 091113. https://doi.org/10.1063/1.4943088.

(15) Giordano, M. C.; Mastel, S.; Liewald, C.; Columbo, L. L.; Brambilla, M.; Viti, L.; Politano, A.; Zhang, K.; Li, L.; Davies, A. G.; et al. Phase-Resolved Terahertz Self-Detection nearField Microscopy. Opt. Express 2018, $26 \quad$ (14), 18423. https://doi.org/10.1364/OE.26.018423. 
(16) Degl'Innocenti, R.; Wallis, R.; Wei, B.; Xiao, L.; Kindness, S. J.; Mitrofanov, O.; Braeuninger-Weimer, P.; Hofmann, S.; Beere, H. E.; Ritchie, D. A. Terahertz Nanoscopy of Plasmonic Resonances with a Quantum Cascade Laser. ACS Photonics 2017, 4 (9), 2150-2157. https://doi.org/10.1021/acsphotonics.7b00687.

(17) Moon, K.; Do, Y.; Lim, M.; Lee, G.; Kang, H.; Park, K.-S.; Han, H. Quantitative Coherent Scattering Spectra in Apertureless Terahertz Pulse Near-Field Microscopes. Appl. Phys. Lett. 2012, 101 (1), 011109. https://doi.org/10.1063/1.4733475.

(18) Moon, K.; Park, H.; Kim, J.; Do, Y.; Lee, S.; Lee, G.; Kang, H.; Han, H. Subsurface Nanoimaging by Broadband Terahertz Pulse Near-Field Microscopy. Nano Lett. 2015, 15 (1), 549-552. https://doi.org/10.1021/nl503998v.

(19) Ropers, C.; Neacsu, C. C.; Elsaesser, T.; Albrechty, M.; Raschke, M. B.; Lienau, C. GratingCoupling of Surface Plasmons onto Metallic Tips: A Nanoconfined Light Source. Nano Lett. 2007, 7 (9), 2784-2788. https://doi.org/10.1021/n1071340m.

(20) De Angelis, F.; Das, G.; Candeloro, P.; Patrini, M.; Galli, M.; Bek, A.; Lazzarino, M.; Maksymov, I.; Liberale, C.; Andreani, L. C.; et al. Nanoscale Chemical Mapping Using Three-Dimensional Adiabatic Compression of Surface Plasmon Polaritons. Nat. Nanotechnol. 2010, 5 (1), 67-72. https://doi.org/10.1038/nnano.2009.348.

(21) Siday, T.; Natrella, M.; Wu, J.; Liu, H.; Mitrofanov, O. Resonant Terahertz Probes for NearField Scattering Microscopy. Opt. Express 2017, 25 (22), 27874. https://doi.org/10.1364/OE.25.027874.

(22) Huth, F.; Chuvilin, A.; Schnell, M.; Amenabar, I.; Krutokhvostov, R.; Lopatin, S.; Hillenbrand, R. Resonant Antenna Probes for Tip-Enhanced Infrared Near-Field Microscopy. Nano Lett. 2013, 13 (3), 1065-1072. https://doi.org/10.1021/nl304289g.

(23) Mastel, S.; Lundeberg, M. B.; Alonso-González, P.; Gao, Y.; Watanabe, K.; Taniguchi, T.; Hone, J.; Koppens, F. H. L.; Nikitin, A. Y.; Hillenbrand, R. Terahertz Nanofocusing with Cantilevered Terahertz-Resonant Antenna Tips. Nano Lett. 2017, 17 (11), 6526-6533. https://doi.org/10.1021/acs.nanolett.7b01924. 
(24) Giessibl, F. J. Advances in Atomic Force Microscopy. Rev. Mod. Phys. 2003, 75 (3), 949 983. https://doi.org/10.1103/RevModPhys.75.949.

(25) García, R. Dynamic Atomic Force Microscopy Methods. Surf. Sci. Rep. 2002, 47 (6-8), 197-301. https://doi.org/10.1016/S0167-5729(02)00077-8.

5 (26) Maghelli, N.; Labardi, M.; Patane, S.; Irrera, F.; Allegrini, M. Optical Near-Field Harmonic Demodulation in Apertureless Microscopy. J. Microsc. 2001, 202 (1), 84-93. https://doi.org/10.1046/j.1365-2818.2001.00882.x.

(27) Mitrofanov, O.; Brener, I.; Luk, T. S.; Reno, J. L. Photoconductive Terahertz Near-Field Detector with a Hybrid Nanoantenna Array Cavity. ACS Photonics 2015, 2 (12), $1763-$ 1768. https://doi.org/10.1021/acsphotonics.5b00475.

11 (28) Balanis, C. A. Antenna Theory: Analysis and Design; John wiley \& sons, 2016.

12 (29) Aghamiri, N. A.; Huth, F.; Huber, A. J.; Fali, A.; Hillenbrand, R.; Abate, Y.; Abate, Y. 13 Hyperspectral Time-Domain Terahertz Nano-Imaging. Opt. Express 2019, 27 (17), 24231. 14 https://doi.org/10.1364/OE.27.024231. 